\title{
De la toile d'araignée... au piège à brouillard
}

\author{
Jean Bouloc \\ Ingénieur hydraulicien - Consultant pour le PNUD et la CCE
}

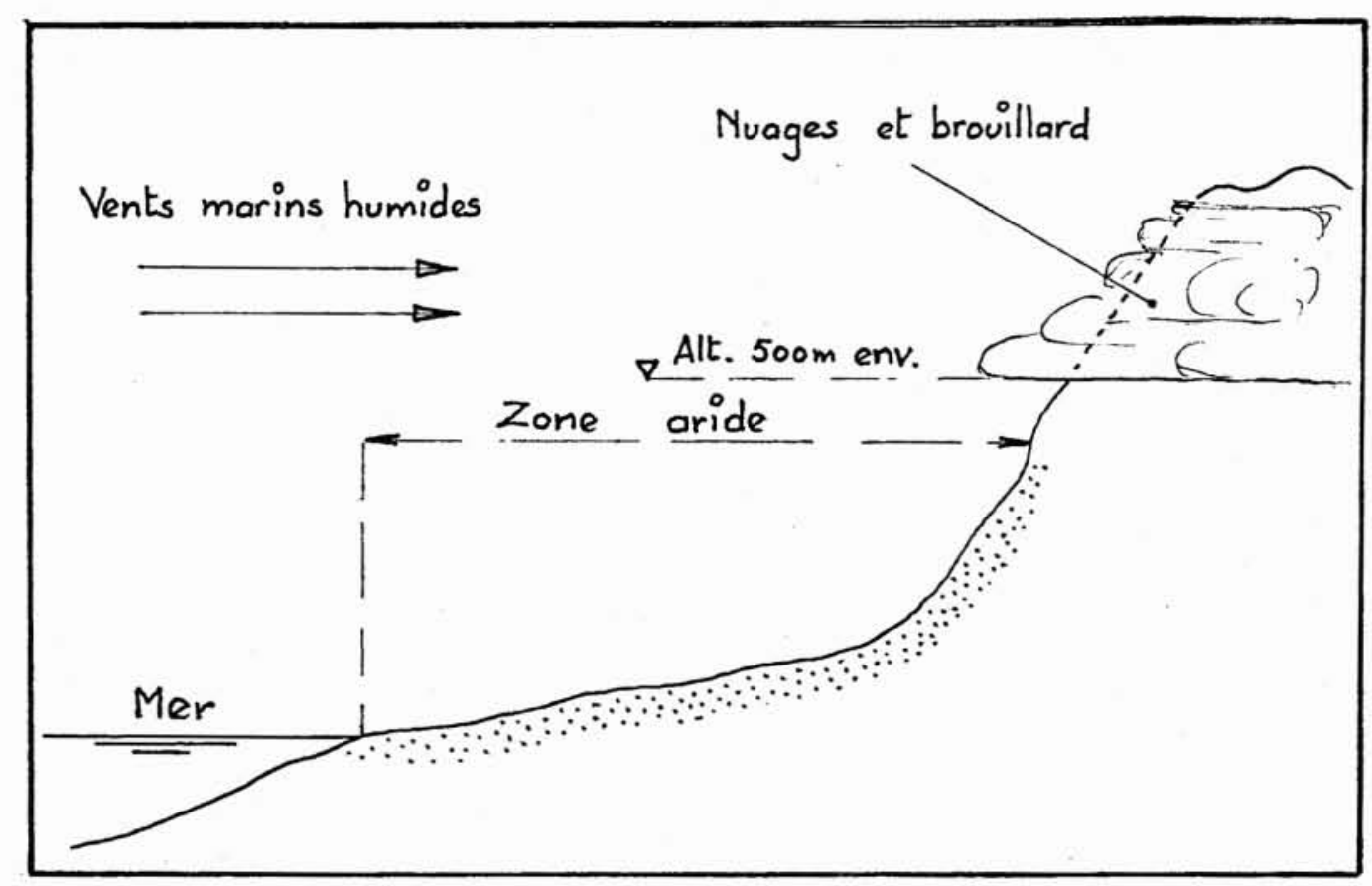

1. Régions favorables à la condensation.

\section{Régions côtières arides}

En plusieurs endroits du globe s'étendent d'immenses zones semi-désertiques, paradoxalement situées en bordure de mer. Lorsque ces régions sont limitées, à l'intérieur des terres, par des chaînes montagneuses̉, les vents marins humides franchissent les zones côtières basses sans provoquer de précipitations et vont alimenter un système nuageux sur les reliefs (fig. I).

Ces nuages, de type "stratus basse altitude ", enveloppent les sommets, où ils forment une mer de brouillard quasi-permanente entre les altitudes 500 et $1000 \mathrm{~m}$ environ. Mais les pluies y sont très rares.

La carte de la figure 2 (page suivante) montre les régions, où l'on rencontre ces caractéristiques topographiques et climatiques. Les zones les plus typiques se trouvent situées principalement sur :

- la côte pacifique du Chili et du Pérou,

- les Îles Canaries et du Cap-Vert,

- certaines côtes d’Afrique (Namibie, Éthiopie). 

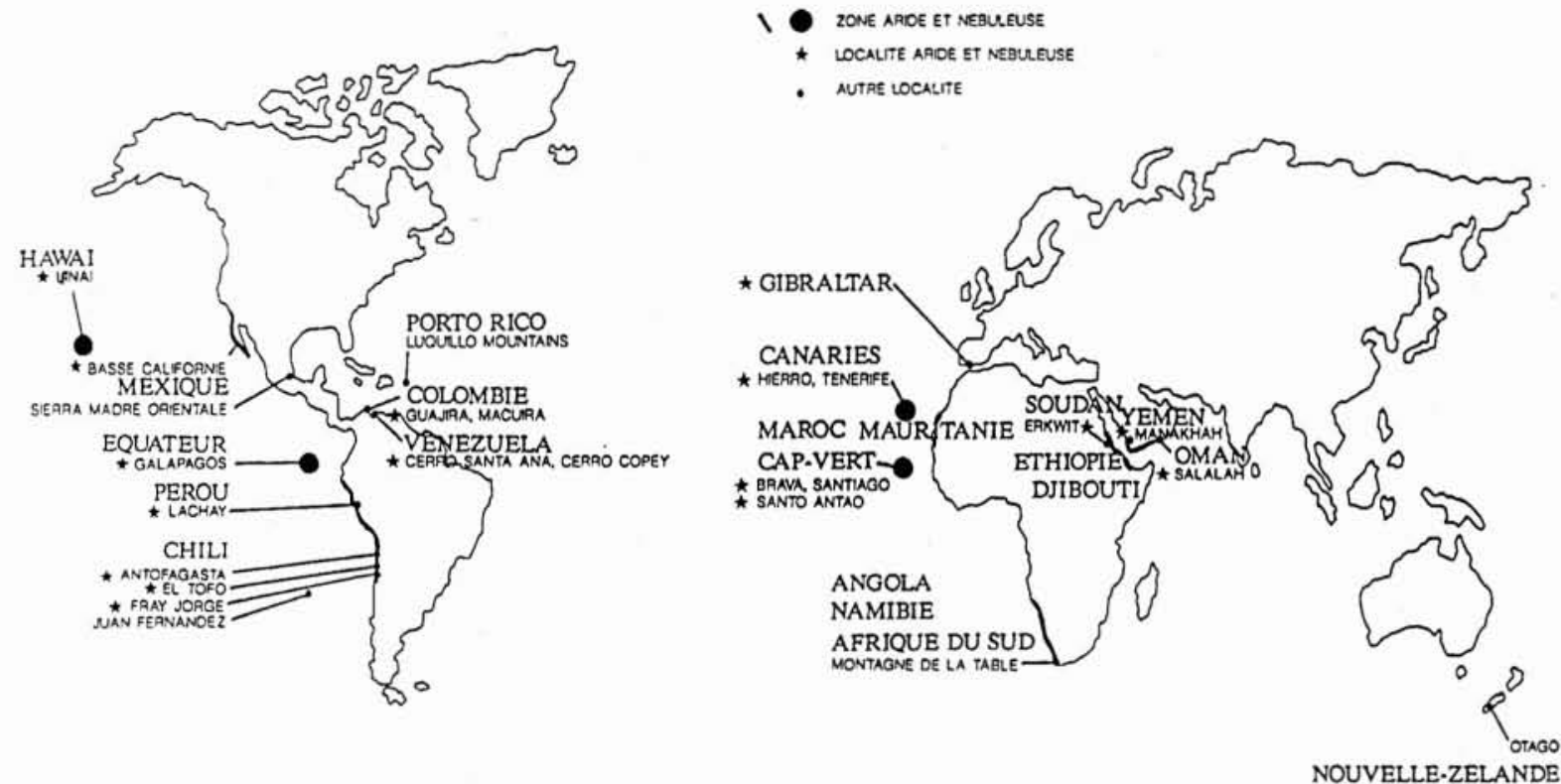

2. Brouillards et zones arides tropicales (la Recherche, déc. 1992).

\section{Principales méthodes de condensation des brouil- lards}

Ces masses nuageuses constituent une énorme ressource hydrique et, très tôt, l'Homme habitant ces zones arides a essayé de capter cette eau. En observant la nature, les 3 règnes (minéral, végétal et animal) lui donnèrent des idées :

\section{- Règne minéral}

Des blocs de certains types de roches calcaires, accumulés en tas, présentent la particularité de pouvoir condenser le brouillard dans les interstices. Il suffit de disposer sous le tas une surface imperméable pour recueillir l'eau condensée.

Il semble que cette méthode était connue dès l'Antiquité [1]. Avant la seconde guerre mondiale, quelques recherches furent effectuées dans ce domaine en Europe. En France même, un dispositif similaire appelé " puits aérien " avait été construit et expérimenté vers 1930 à Trans-enProvence, mais son fonctionnement ne fût pas couronné de succès : il a été abandonné.

Cette technique de condensation des brouillards, que l'on rencontre encore, de manière très rustique, dans certaines régions montagneuses semi-désertiques d'Afrique, ne semble pas, à notre connaissance, donner lieu actuellement à des recherches poussées.

- Règne végétal

Par contre, on a constaté depuis très longtemps que certaines plantes et arbres peuvent pousser très aisément dans des zones à précipitation presque nulle, uniquement en captant l'humidité des brouillards, et même en produisant de l'eau.

A. Acosta Baladon signale que, dès le $16^{\mathrm{e}}$ siècle, on rencontrait aux Îles Canaries un arbre dénommé "garoé ". Cet " arbre-fontaine " captait l'eau des brouillards et fournissait une eau douce en abondance. D'autres espèces telles que caféier, eucalyptus, dattier, acacia, présentent la même particularité. Certains types d'agaves fournissent jusqu'à 20 litres d'eau par jour au Cap-Vert pendant les périodes de brouillard, qui durent souvent plus de 200 jours par an [2 et 3].

Sur cette question, des études très complexes sont menées dans divers centres de recherches et, en particulier, à l'ORSTOM et au Centre de coopération internationale en recherche agronomique pour le développement (CIRAD) à Montpellier, en vue de sélectionner des espèces végétales qui seraient employées pour le reboissement de régions arides.

\section{- Règne animal}

Mais c'est surtout l'observation du règne animal et, plus particulièrement, la toile que tisse l'araignée, qui a donné à l'Homme l'idée d'utiliser une technique similaire.

On constate en effet que, par temps de brouillard, les toiles d'araignées se couvrent de fines gouttelettes qui grossissent peu à peu par coalescence. Pourquoi ne pas tendre de grands filets, qui permettraient, sur le même principe, de condenser l'eau des brouillards? 


\section{Filets capteurs de brouillards}

\subsection{Bref rappel historique}

Les recherches dans ce domaine particulier sont assez récentes puisqu'il semble que HoHENPEISSENBERG ait été le premier à essayer en 1950 en Allemagne un capteur expérimental de petite dimension en toile métallique.

Des améliorations furent ensuite apportées à ce dispositif par plusieurs chercheurs en Afrique du Sud (1955), en Australie (1956). En 1957-58, HurST utilisa à Gibraltar un grillage galvanisé, type moustiquaire, qui a donné des résultats prometteurs.

À la même époque, des techniciens de l'Université du Nord à Antofagasta (Chili) firent des essais de capteurs avec des systèmes maillés en nylon.

Également, le Portugal s'intéressa à ce procédé pour les Îles du Cap-Vert, qui présentent des caractéristiques climatiques particulièrement favorables. C'est F. ReIS CUNHA qui réalisa, à partir de 1961, des recherches sur la condensation d'abord en laboratoire, puis sur les îles, en comparant les rendements de divers types de matériau constituant le capteur (grillage métallique, moustiquaire, filet plastique tendu sur un cadre), et en faisant varier ses caractéristiques (situation et inclinaison du capteur, maillage, trame, filet simple ou double, etc...). Les volumes d'eau recueillis atteignirent parfois 10 et 20 litres par $\mathrm{m}^{2}$ de filet et par jour : valeurs très encourageantes qui permirent à F. REIS CUNHA d'indiquer sur ces îles les zones les plus propices [4]. Les capteurs utilisés furent ensuite perfectionnés par A. ACOSTA BALADON.

La figure 3 montre une station expérimentale en service a Santiago (Cap-Vert).

\subsection{Application en Amérique Latine}

Les excellents résultats obtenus en phase expérimentale incitèrent plusieurs pays à se lancer dans une exploitation à plus grande échelle. Le Chili et le Pérou sont certainement parmi les plus avancés dans cette technique et possèdent déjà des installations très spectaculaires.

\subsubsection{Chili}

Dans ce pays, R.S. Schemenauer, d'Environnement Canada, et Pilar CERECEDA, de l'Université catholique du Chili à Santiago [5]. bénéficiant de subventions canadiennes, sont à l'origine de plusieurs réalisations, dont celle de El Tofo (1987-1989).

Cette installation, une des plus importantes, comprend :

- 75 panneaux de $48 \mathrm{~m}^{2}$ chacun $(4 \times 12)$, soit au total $3600 \mathrm{~m}^{2}$. Ces panneaux sont constitués par un filet en fibre polypropylène (maille de 3 à $5 \mathrm{~mm}$ ) tendu sur un cadre. Ils sont plantés verticalement sur une crête montagneuse, à $800 \mathrm{~m}$ d'altitude.

- Une goulotte à la partie inférieure de chaque panneau recueille les gouttes.

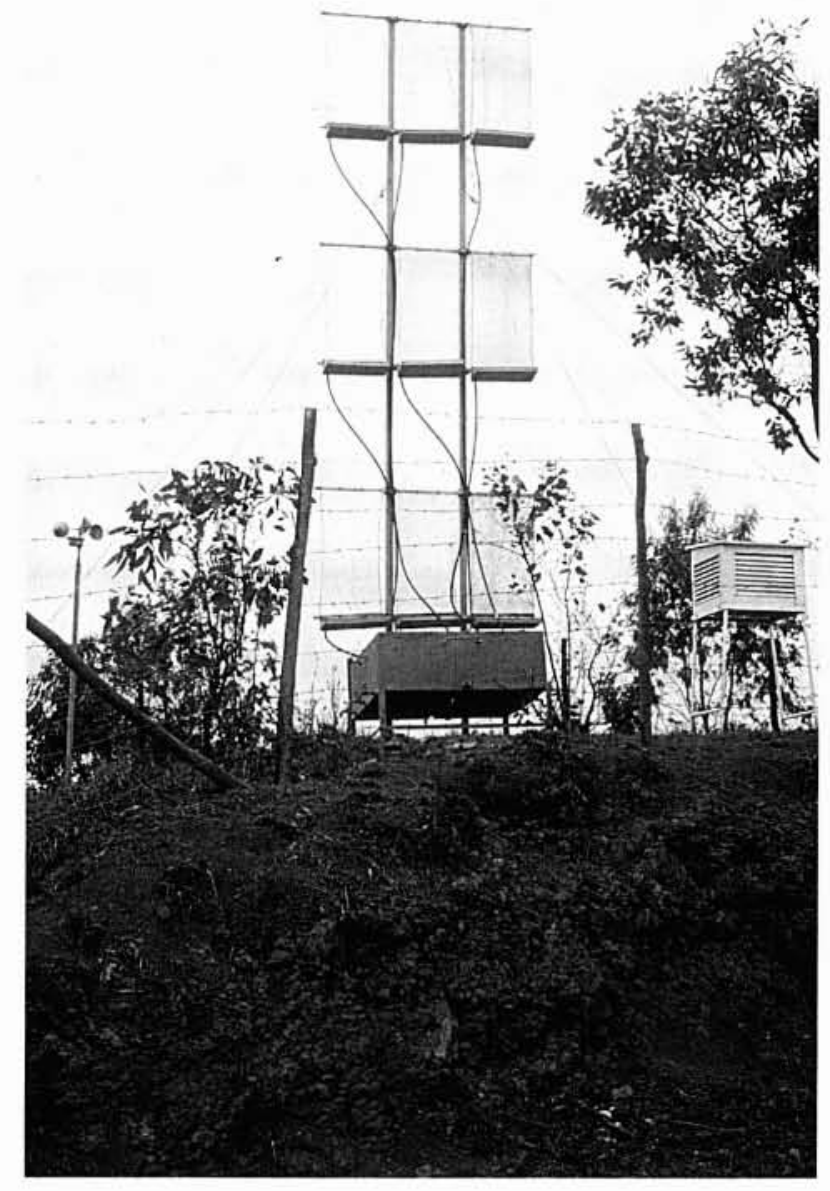

3. Station expérimentale à Santiago (Îles du Cap-Vert).

- Une conduite en PVC amène l'eau par gravité à 2 réservoirs de 25 et $100 \mathrm{~m}^{3}$.

L'eau collectée sert à alimenter le village de Chungungo (330 habitants) situé en pied de montagne et à l'arrosage de parcelles reboisées.

Le débit de l'installation, mesuré sur les 50 premiers panneaux, s'est élevé à $72001 /$ jour en moyenne sur trois ans, soit :

$$
\frac{7200}{50 \times 48}=31 / \mathrm{j} / \mathrm{m}^{2} \text { de filet }
$$

Cette valeur doit être considérée comme très modeste, car ces trois premières années ont été particulièrement sèches, la pluviométrie annuelle n'ayant été que de 10, 50, $10 \mathrm{~mm}$, alors que la valeur normale est de $80 \mathrm{~mm}$. On peut donc espérer collecter en année moyenne des volumes d'eau nettement supérieurs. 
J. BOULOC

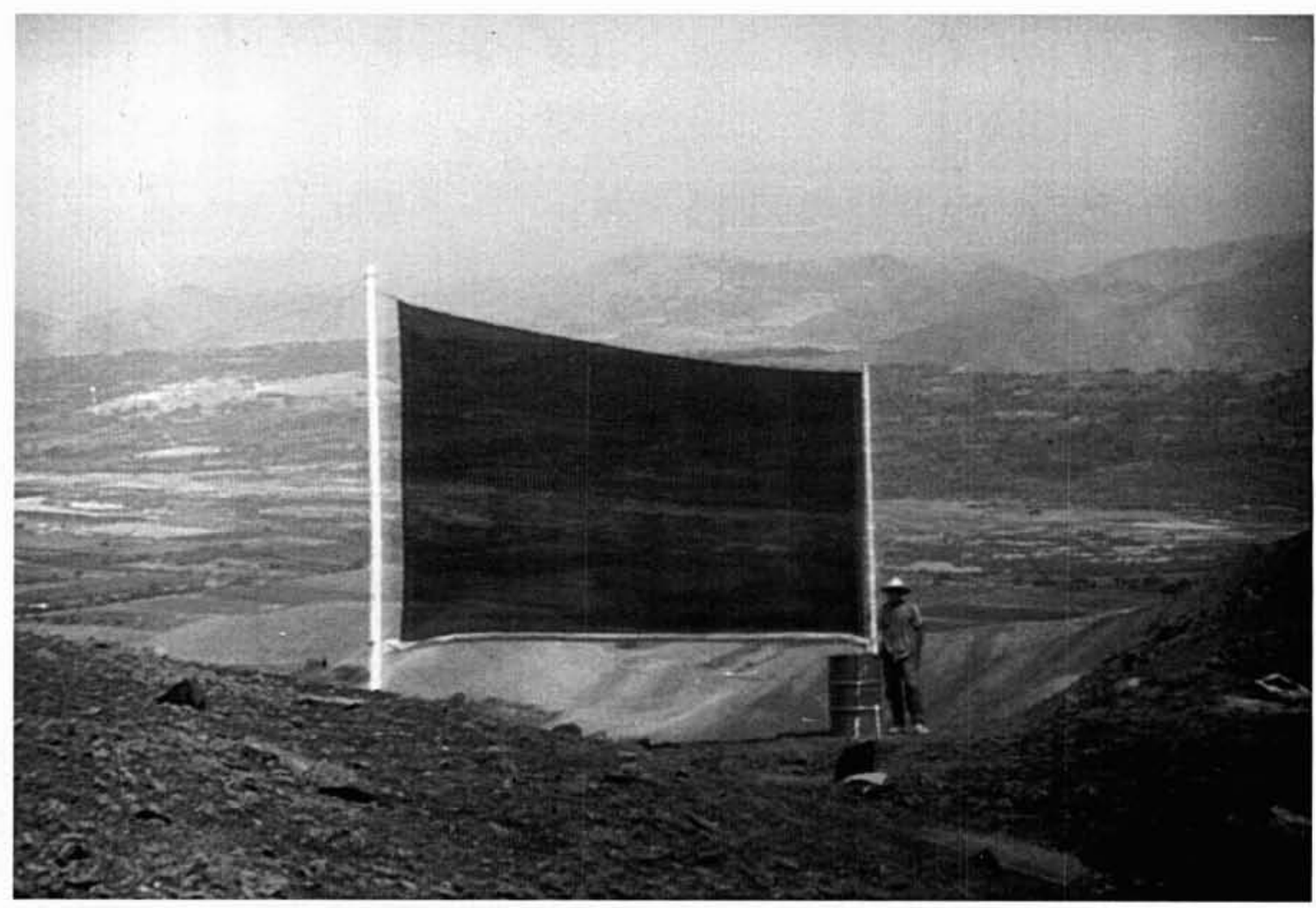

4. Capteur expérimental près de Lima.

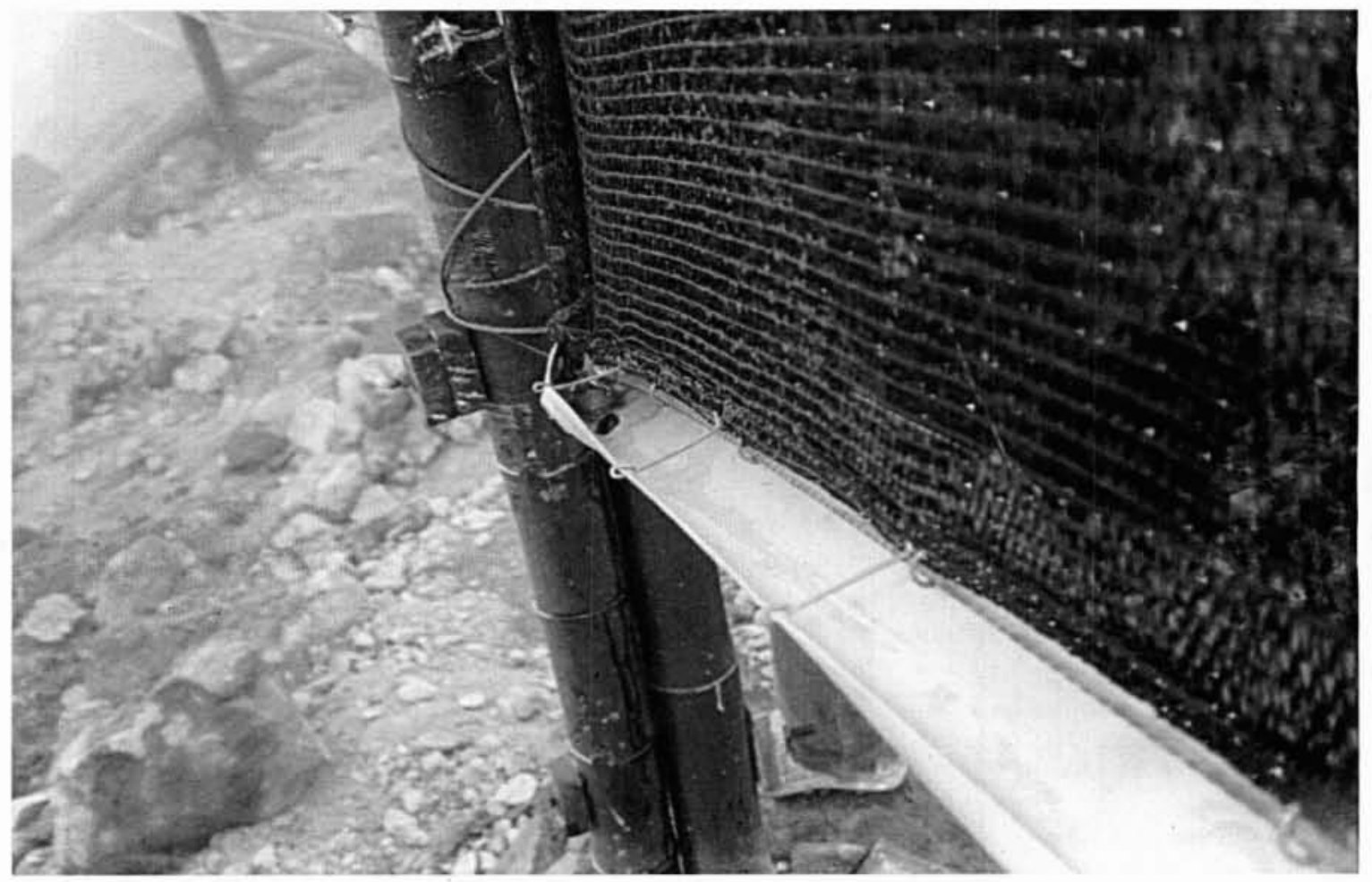

5. Vue de la goulotte qui recueille l'eau condensée. 


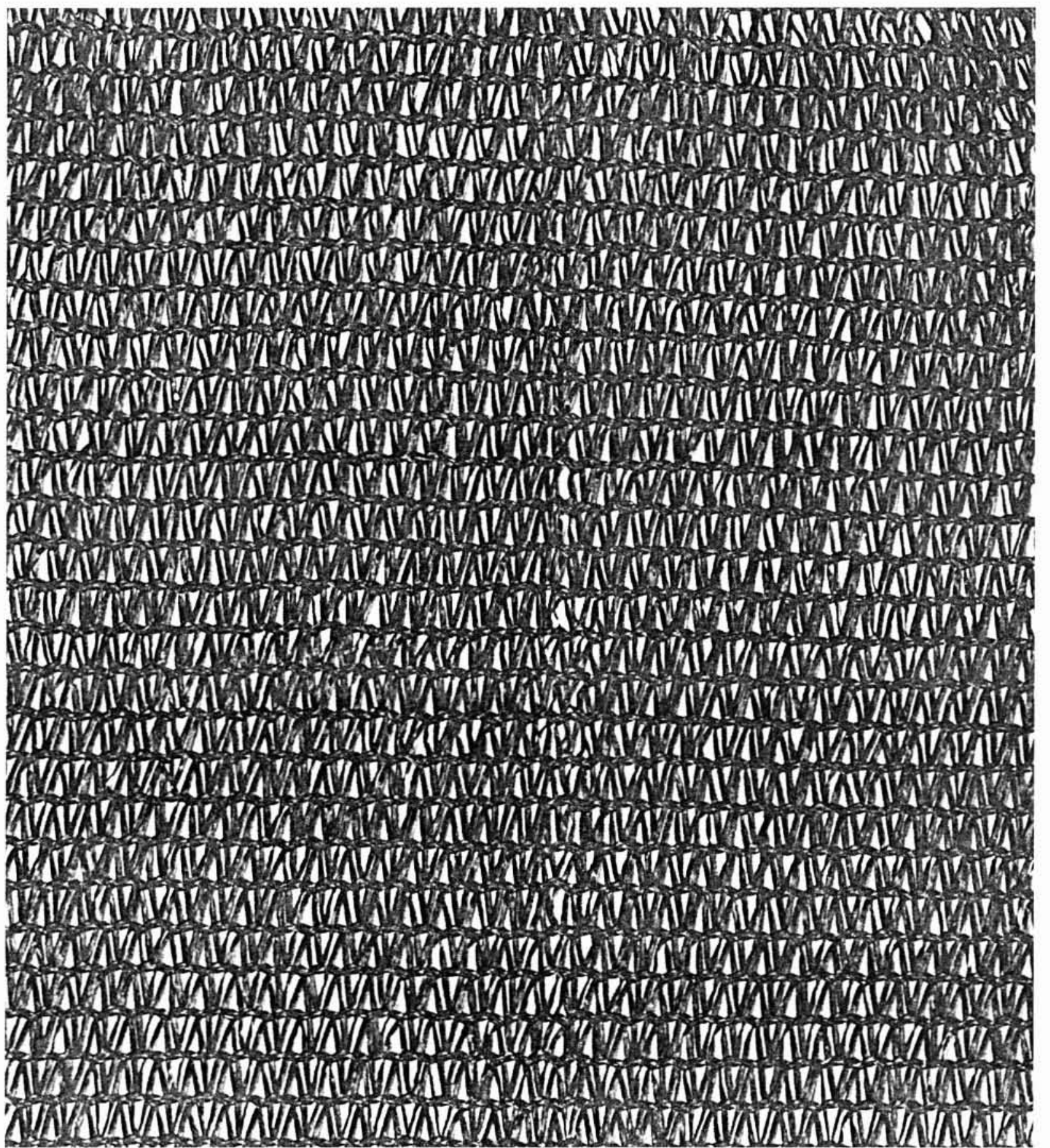

6. Filkt en polyproprlènc uilisé au Chili et au Pérou (èchelle l

3.2.2 Pérou

Le Pérou possède la même bande côtière aride, et par conséquent des possibilités de captage d'eau des brouillards identiques à celles du Chili (fig. 4 et 5 ).
Bénéficiant de l'expérience chilienne [6]. et grâce ả une collaboration française, plusieurs installations fonctionnent avec succès. C'est le Service national de météorologie et d'hydrologie (SENAMHI) qui exploite ce procédé, et la société Estratus S.R.L. fournit un filet en polypropylène fabriqué en France (fig. 6 ) 


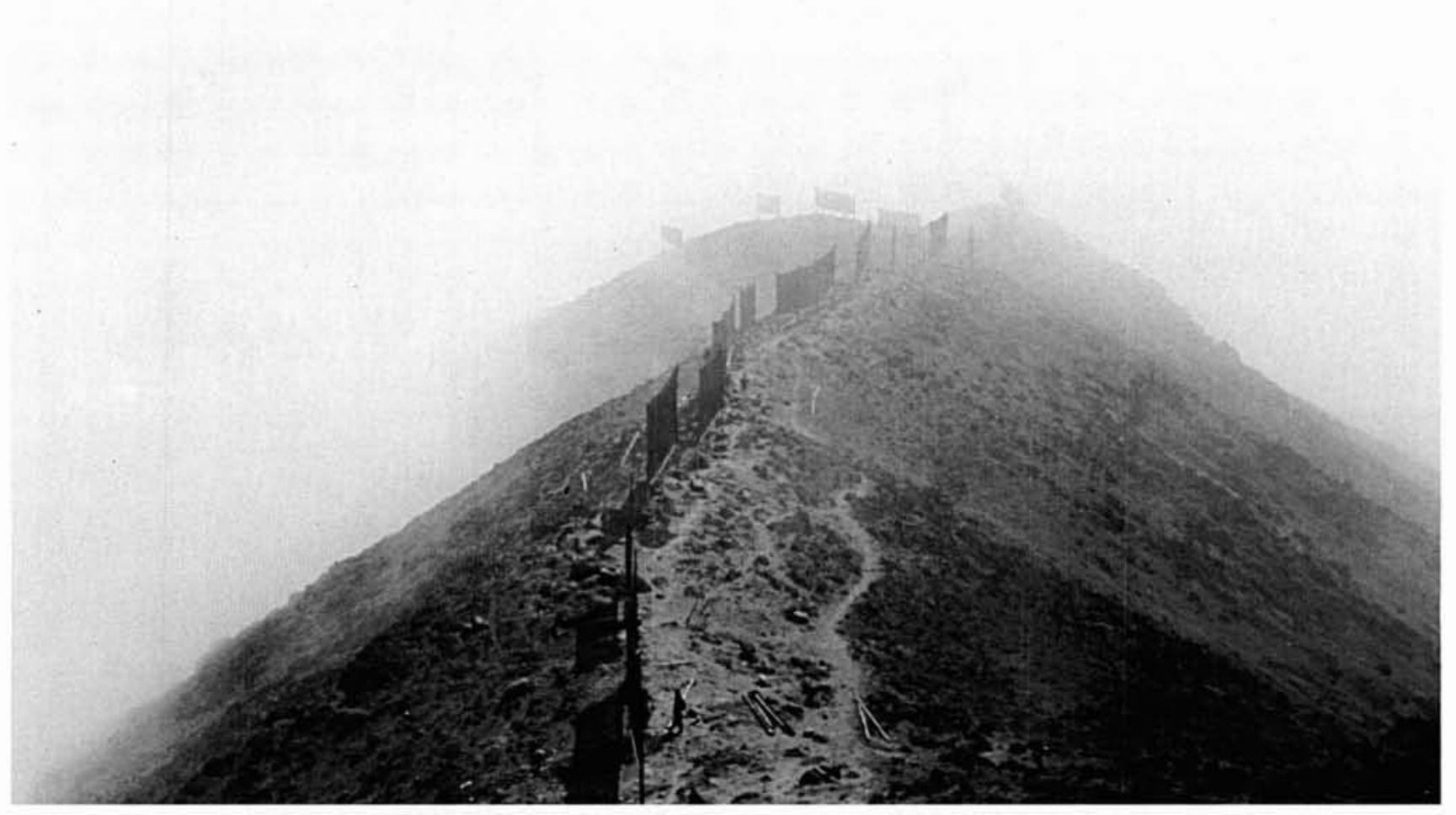

7. Situation des panneaux en crête de la montagne, dans le brouillard.

Parmi les diverses installations, existantes ou en projet, on peut citer :

- Le collège Villa Caritas à La Molina (fig. 7, 8 et 9) Ce collège reçoit l'eau captée par une rangée de 28 panneaux (surface totale : $2500 \mathrm{~m}^{2}$ ) implantés à $750 \mathrm{~m}$ d'altitude, et répartis sur une distance de $1200 \mathrm{~m}$. Le filet est de même nature que celui utilisé au Chili.

Le volume moyen journalier récolté est de 10000 litres, ce qui donne un rendement spécifique de $4 \mathrm{l} / \mathrm{m}^{2}$ de filet, pouvant parfois atteindre 30 et $40 \mathrm{l} / \mathrm{m}^{2} /$ jour par temps de brouillard très épais, c'est-à-dire 10 fois plus.

Une conduite en PVC de $1300 \mathrm{~m}$ de longueur amène l'eau au réservoir du collège.

Un projet, en cours d'étude, prévoit de doubler la surface de captage de cette installation.

- Les Rosales à Ventanilla

Après des essais très productifs sur une installation réduite de $16 \times 2=32 \mathrm{~m}^{2}$, on projette d'implanter
$5000 \mathrm{~m}^{2}$ de capteurs identiques à ceux de Villa Caritas (projet Cerro Orara).

La production prévue est de 40 à $200 \mathrm{~m}^{3} /$ jour pour desservir une agglomération de 3000 habitants, ce qui donnerait entre 10 et $60 \mathrm{l} / \mathrm{j} / \mathrm{h}$.

\subsubsection{Application à l'agriculture}

Le même principe de la " toile d'araignée " est appliquée à une très petite échelle, au Pérou comme au Chili, pour alimenter en eau certaines plantations : figuiers de barbarie, jeunes arbustes pour le reboisement, par exemple.

Chaque plant est protégé par un capteur individuel en forme de raquette tendant un filet en nylon et fiché verticalement en terre, à côté. Les gouttes collectées parviennent ainsi directement au sol. Parfois même, un procédé plus rudimentaire consiste à enfoncer 2 piquets près de la plante et à tendre entre-eux un morceau de... bas nylon! Quel ingénieux système d'irrigation au goutte à goutte! 


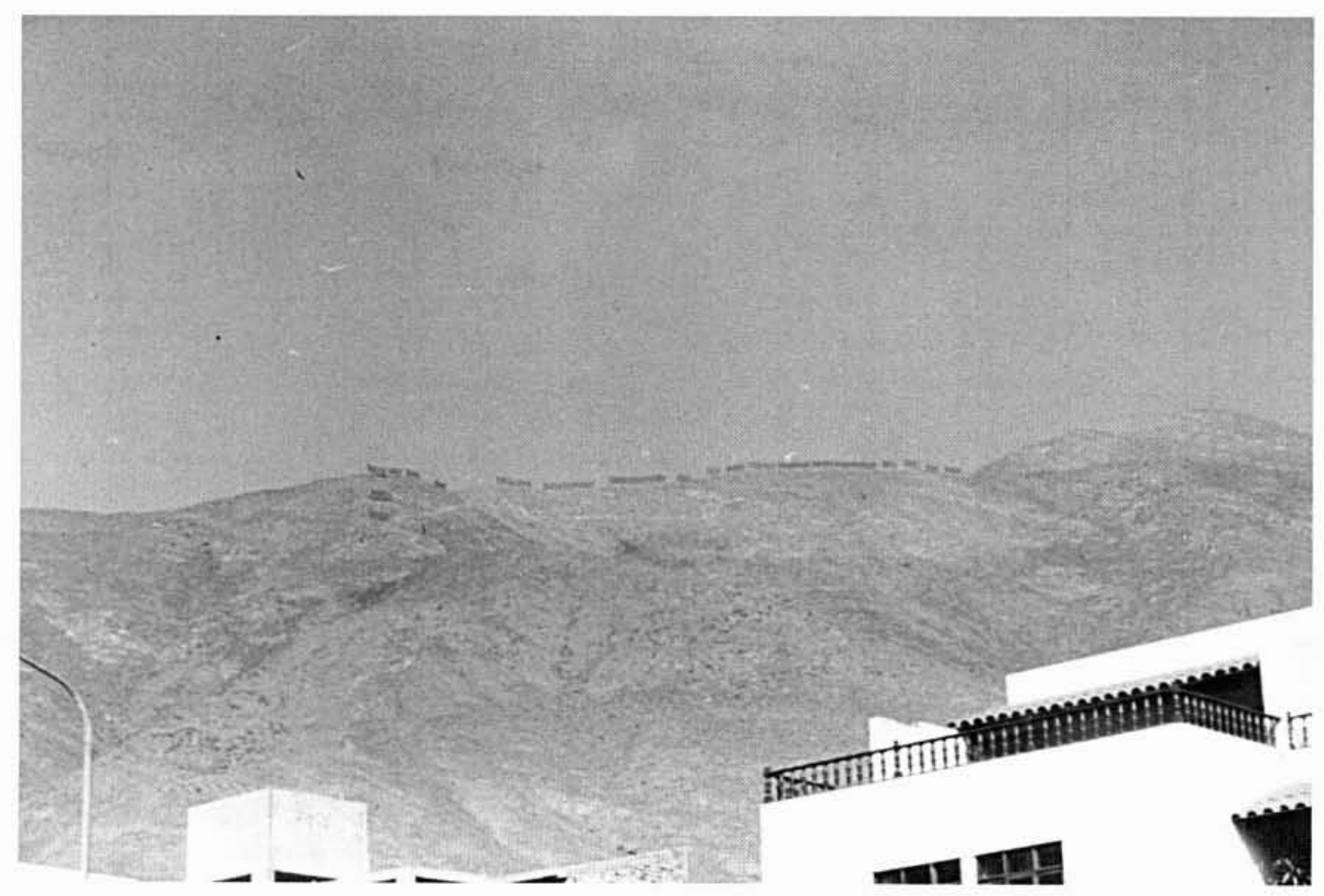

8. Vue des panneaux depuis le Collège (volume d'eau recueil$l i=10000$ litres (iour).

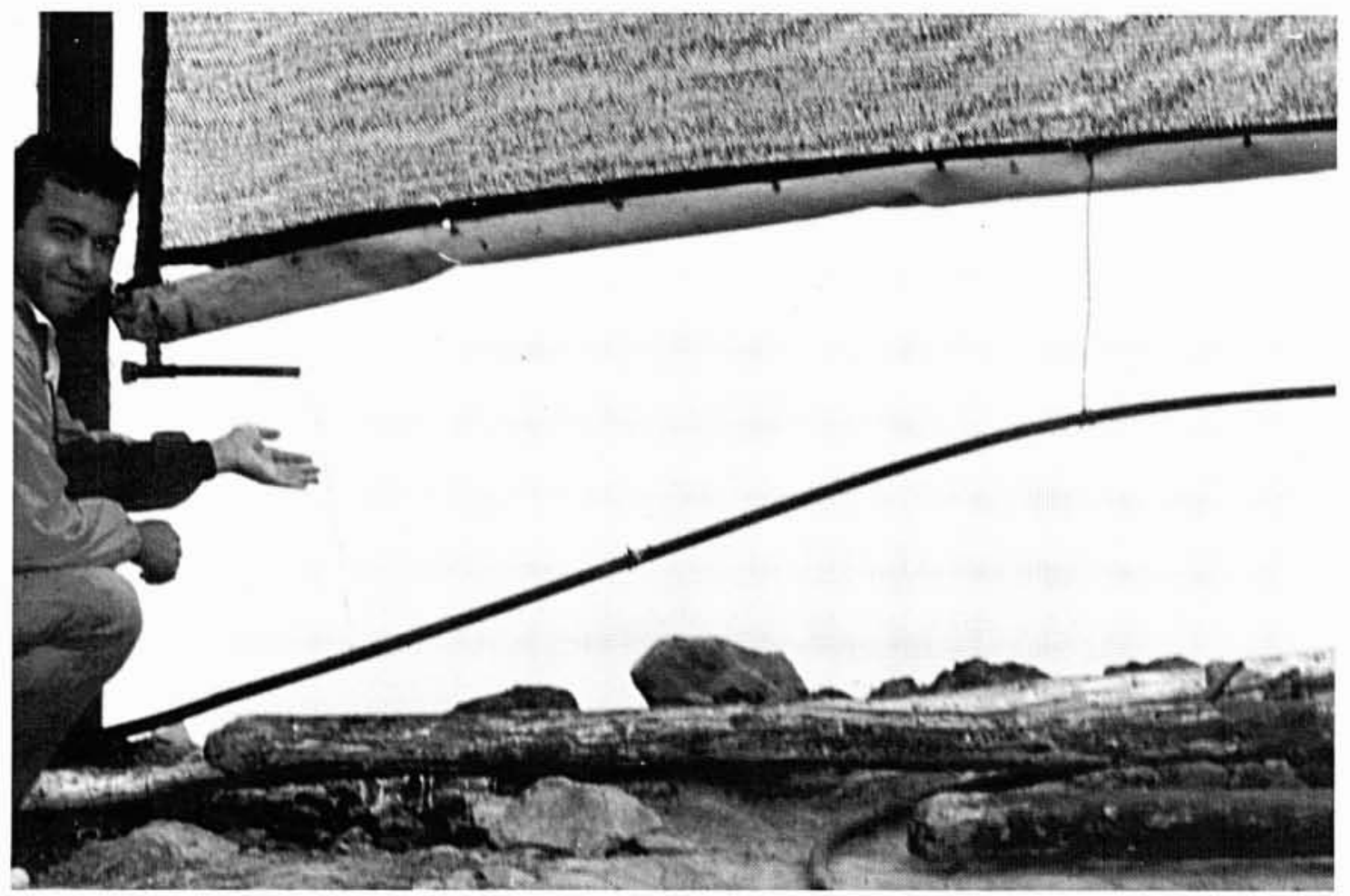

9. On distingue le filet d'eau s'écoulant de la goulotte d'un panneau de $50 \mathrm{~m}^{2}$. 


\section{Intérêt et avantages de ce procédé}

Ce procédé, lorsqu'il est applicable, présente des caractéristiques particulièrement intéressantes pour alimenter en eau des populations de régions semi-désertiques, où tout autre solution serait très coûteuse.

4.1. Ressource en eau toujours renouvelée : Les nuages sont amenés par les vents humides soufflant de la mer presque constamment. Et, de plus, on pense que les capteurs ne prélèvent que quelques $\%$ du volume d'eau contenue en suspension dans le brouillard intercepté par les cadres.

4.2. Aucune énergie : L'exploitation du dispositif ne nécessite aucune énergie, puisque l'eau s'écoule ensuite par gravité jusqu'au lieu d'utilisation.

4.3. Installation et entretien aisés: Les panneaux sont généralement fixés sur des poteaux en bois.

Le filet et les tuyaux en PVC sont, jusqu'à ce jour, importés. Dans le cas du Pérou, ils sont offerts par une coopération française.

L'implantation du système peut être effectuée par du personnel local encadré, et la maintenance, très simple, est sous la responsabilité de la population bénéficiaire.

Enfin, il faut signaler que la société Estratus réalise, à Lima, de petits capteurs standards de 1 ou $2 \mathrm{~m}^{2}$ constitués d'un filet tendu sur un cadre rectangulaire. Ces capteurs sont fournis aux particuliers ou organismes intéressés, afin de voir si un site est favorable à la condensation et mérite une installation plus importante.

4.4. Eau de bonne qualité: Généralement, cette eau est parfaitement potable, mais des analyses très sérieuses se poursuivent au Chili pour connaître avec précision ses caractéristiques [7].

Le $\mathrm{pH}$ est parfois faible, entre 5 et 7 . Mais il paraît acceptable car il n'y a pas de risque de corrosion des tuyaux. Les concentrations en ions restent bien en dessous des limites OMS.

Néanmoins, on a parfois remarqué que, pour certains points du Pérou, l'eau recueillie présente une légère pollution d'origine urbaine et industrielle, lorsque les vents porteurs d'humidité passent au dessus de la ville de Lima.

Enfin, un risque de contamination pourrait provenir des goulottes collectrices à l'air libre, qui doivent être maintenues propres. De plus, elles sont toujours situées à 1 ou $2 \mathrm{~m}$ au dessus du sol, de façon à être hors de portée des animaux.

4.5. Faible prix de revient : Nous n'avons pas eu connaissance des calculs du coût d'installation et de fonctionnement de ces dispositifs. Ce coût dépend de nombreux paramètres (main-d'œuvre, site et accessibilité, type et fourniture du matériel, etc...), qui varient fortement d'un site à un autre.

Une estimation grossière faite au Chili conduit à un prix de revient de 1 US $\$ / \mathrm{m}^{3}$ environ pour une consommation près des capteurs, à 2 US $\$ / \mathrm{m}^{3}$ si le village est à $6 \mathrm{~km}$. Ce prix est à comparer à celui d'une adduction classique, qui conduirait à un coût de l'ordre de 6 à 8 US $\$ / \mathrm{m}^{3}$.

\section{Conclusion}

Dans divers pays des recherches se poursuivent pour essayer d'améliorer ce dernier procédé et le rendre plus performant: matériau nouveau, panneaux orientables, filets doubles parallèles, afin que les gouttes arrachées du premier en cas de fort vent soient récupérées par le deuxième, etc... Et l'imagination de l'Homme étant sans bornes, on envisage même une gigantesque toile d'araignée en nylon, qui pourrait être tendue par des câbles, en travers d'un col de montagne. Un grand constructeur français de téléphérique réfléchirait au problème...

Nous terminerons cet exposé en citant A. Acosta BALADON, agrométéorologue, expert de l'OMM, qui écrivait récemment :

"Ces précipitations occultes constituent une ressource naturelle renouvelable très importante et capable de résoudre à un coût réduit, de nombreux problèmes posés, soit pour des aménagements agricoles, soit pour de nouvelles installations de populations dans de vastes régions du monde, en leur fournissant une eau potable, comme cela est possible le long de la côte pacifique du Chili et du Pérou entre les parallèles 8 et $32^{\circ}$ de latitude Sud, le désert de Namibie, les Îles du Cap-Vert, Oman, Yemen, etc...”

\section{Bibliographie sommaire}

[1] Alain Gioda (ORSTOM), A. Acosta Baladon (OMM). "Les puits aériens de Theodosia, de Montpellier et de Trans ". Sécheresse, $\mathrm{n}^{\circ} 3$, vol. 2, septembre 1991.

[2] A. Acosta Baladon (OMM), Alain Gioda (ORSTOM). "L'importance des précipitations occultes sous les tropiques secs. Essai de synthèse ". VII Congrès Mondial des Ressources en Eau, Rabat, Maroc, mai 1991.

[3] A. Gioda, A. Acosta Baladon, P. Fontanel, Z. Martin Hernandez, A. Santos. - "L'arbre-fontaine". La Recherche, vol. 23, décembre 1992.

[4] F. Reis Cunha. - "O problema da captação da água do nevoeiro em Cabo Verde ". Estudos Agronomicos do Ultramar. Lisboa, Portugal, 1964.

[5] R. S. Schemenauer et P. Cereceda. - " The collection of fog water in Chile for use in Coastal Villages ". VIth IWRA World Congress, Ottawa, Canada, 1988.

[6] R.S. Schemenauer, P. Cereceda, M. Suit. - "Water production at a coastal fog collection site in Peru n. Atmosfera, 1992.

[7] R. S. Schemenauer, P. Cereceda. — " The chemical composition of fog water supplies in rural areas of Chile, Peru and Oman ". International Seminar on Efficient Water Use, Mexico City, Mexico, 1991 